\title{
Josephson critical current in a long mesoscopic S-N-S junction
}

\author{
P. Dubos, ${ }^{1}$ H. Courtois, ${ }^{1}$ B. Pannetier, ${ }^{1}$ F. K. Wilhelm, ${ }^{2,3}$ A. D. Zaikin, ${ }^{4}$ and G. Schön ${ }^{2}$ \\ ${ }^{1}$ Centre de Recherches sur les Très Basses Températures-C.N.R.S. associated to Université Joseph Fourier, 25 Avenue des Martyrs, \\ 38042 Grenoble, France \\ ${ }^{2}$ Institut für Theoretische Festkörperphysik, Universität Karlsruhe, D-76128 Karlsruhe, Germany \\ ${ }^{3}$ Quantum Transport Group, Department of Applied Physics and DIMES, TU Delft, 2600 GA Delft, The Netherlands \\ ${ }^{4}$ Forschungszentrum Karlsruhe, Institut für Nanotechnologie, D-76021 Karlsruhe, Germany
}

(Received 10 August 2000; published 18 January 2001)

\begin{abstract}
We carry out an extensive experimental and theoretical study of the Josephson effect in S-N-S junctions made of a diffusive normal metal $(\mathrm{N})$ embedded between two superconducting electrodes $(\mathrm{S})$. Our experiments are performed on $\mathrm{Nb}-\mathrm{Cu}-\mathrm{Nb}$ junctions with highly transparent interfaces. We give the predictions of the quasiclassical theory in various regimes on a precise and quantitative level. We describe the crossover between the short- and the long-junction regimes and provide the temperature dependence of the critical current using dimensionless units $e R_{\mathrm{N}} I_{c} / \epsilon_{c}$ and $k_{B} T / \epsilon_{c}$, where $\epsilon_{c}$ is the Thouless energy. Experimental and theoretical results are in excellent quantitative agreement.
\end{abstract}

DOI: 10.1103/PhysRevB.63.064502 PACS number(s): 73.23.-b, 74.50.+r, 74.80.Fp, 85.25.Cp

The Josephson effect is well known to exist in weak links connecting two superconducting electrodes $\mathrm{S}$, e.g., a tunnel barrier I, a short constriction $\mathrm{C}$ or a normal metal N (S-I-S, $\mathrm{S}-\mathrm{C}-\mathrm{S}$, and S-N-S junctions). This effect manifests itself in a nondissipative dc current flowing through the Josephson junction at zero voltage. At weak coupling, e.g., in the S-I-S case, the Josephson current can be expressed as $I_{s}=I_{c} \sin \varphi$, where $\varphi$ is the phase difference between the two superconducting condensates and the maximum supercurrent $I_{c}$ is called the critical current.

The Josephson effect in S-N-S junctions has been studied in a variety of configurations. The early experiments of Clarke $^{1}$ and Shepherd ${ }^{2}$ were performed in $\mathrm{Pb}-\mathrm{Cu}-\mathrm{Pb}$ sandwiches. In these experiments and in the pioneering calculations by de Gennes, ${ }^{3}$ it was already realized that the presence of a supercurrent in such structures is due to the proximity effect. This can be understood as the generation of superconducting correlations in a normal metal connected to a superconductor, mediated by phase-coherent Andreev reflections at the S-N interfaces. The critical current $I_{c}$ is limited by the "bottleneck' in the center of the N-layer, where the pair amplitude is exponentially small: $I_{c} \propto e^{-L / L_{T}}$. Here, $L_{T}$ $=\sqrt{\hbar D / 2 \pi k_{B} T}$ is the characteristic thermal length in the diffusive limit and $L$ is the length of the junction. These calculations, as well as those by Fink, ${ }^{4}$ analyzed the temperature dependence of $I_{c}$ within the Ginzburg-Landau theory in the vicinity of the superconducting critical temperature $T_{c}$. Later, the critical current of diffusive S-N-S microbridges ${ }^{5,6}$ was successfully described by Likharev ${ }^{7}$ with the aid of the quasiclassical Usadel equations. ${ }^{8}$ In this work, the emphasis was put on the high-temperature regime where the superconducting order parameter is smaller than the thermal energy $\Delta \ll k_{B} T$. A more general study of the Josephson effect in diffusive S-N-S junctions was made in Ref. 9.

More recently, experimental data on long Josephson junctions $^{10}$ showed a surprising temperature dependence, which turned out to be in a strong disagreement with the early theory by de Gennes. These data have been discussed by some of us ${ }^{11}$ within the quasiclassical approach, which we will also use in the present work. Fink ${ }^{12}$ attempted to analyze the data ${ }^{10}$ by means of an extrapolation of the GinzburgLandau theory to low temperatures.

The proximity effect in mesoscopic hybrid structures consisting of normal and superconducting metals attracted a growing interest during recent years. ${ }^{13}$ Here we will consider mesoscopic diffusive S-N-S junctions where the sample length is much larger than the elastic mean free path $l_{e}$ but smaller than the dephasing length $L_{\varphi}: l_{e}<L<L_{\varphi}$. In N-S junctions and Andreev interferometers, we can identifyboth theoretically and experimentally-the natural energy scale for the proximity effect. ${ }^{14,15}$ It is given by the Thouless energy $\epsilon_{c}=\hbar D / L^{2}$. Here $D=v_{F} l_{e} / 3$ is the diffusion constant of the $\mathrm{N}$ metal, $v_{F}$ is the Fermi velocity. In contrast to the energy gap $\Delta$ that is set by the interactions in the superconducting electrodes, the energy scale $\epsilon_{c}$ is a single-electron quantity : $\epsilon_{c} / \hbar$ is merely the diffusion rate across the sample for a single electron. This energy scale remains important in nonequilibrium situations, e.g., if one drives the supercurrent across a S-N-S junction by the injection of a control current in the $\mathrm{N}$ metal. ${ }^{16-18}$

The main purpose of the present paper is to carry out a detailed experimental investigation of the equilibrium supercurrent in relatively long diffusive $\mathrm{S}-\mathrm{N}-\mathrm{S}$ junctions with highly transparent N-S interfaces as well as a quantitative comparison of our data to the theoretical predictions. Here, a long junction means that the junction length $L$ is much bigger than $\sqrt{\hbar D / \Delta}$. This is equivalent to $\Delta \gg \epsilon_{c}$. In order to perform this comparison at all relevant temperatures, we complete the previous studies by providing a rigorous expression for the Josephson critical current at $T \rightarrow 0$, which was not properly evaluated before. Our experimental results are in excellent agreement with theoretical predictions.

As before, 9,11 our theoretical approach is based on the quasiclassical Green's functions in imaginary time. The proximity effect is described by a finite pair amplitude $F$ in the $\mathrm{N}$ metal (see Ref. 19 and references therein). We will assume N-S interfaces to be fully transparent and neglect the suppression of the pair potential $\Delta$ in the $\mathrm{S}$ electrodes near 
the N-S interface. This is appropriate at $T \ll T_{c}$ or if the reservoirs are very massive as compared to the normal metal. Within those bounds, our calculation does not contain further approximations and is, e.g., valid at arbitrary temperature and sample size. We will now proceed by discussing certain limits.

In the high-temperature regime $k_{B} T \gg \epsilon_{c}$ (or, equivalently, $L \gg L_{T}$ ), the solution is well known. In this case the mutual influence of the two superconducting electrodes can be neglected and the Usadel equations can be linearized in the $\mathrm{N}$ metal, except in the vicinity of the $\mathrm{N}-\mathrm{S}$ interfaces. One finds: ${ }^{9}$

$$
e R_{\mathrm{N}} I_{c}=64 \pi k_{B} T \sum_{n=0}^{\infty} \frac{L}{L_{\omega_{n}}} \frac{\Delta^{2} \exp \left(-L / L_{\omega_{n}}\right)}{\left[\omega_{n}+\Omega_{n}+\sqrt{2\left(\Omega^{2}+\omega_{n} \Omega_{n}\right)}\right]^{2}},
$$

where $R_{\mathrm{N}}$ is the N-metal resistance, $\omega_{n}=(2 n+1) \pi k_{B} T$ is the Matsubara frequency, $\Omega_{n}=\sqrt{\Delta^{2}+\omega_{n}^{2}}$ and $L_{\omega_{n}}$ $=\sqrt{\hbar D / 2 \omega_{n}}$. If $T$ is close to the critical temperature of $\mathrm{S}$, the gap is small as compared to the thermal energy : $\Delta \ll k_{B} T$. In this limit, Eq. (1) coincides with the result derived by Likharev.?

At lower temperatures $k_{B} T \lesssim \epsilon_{c}$ evaluation of $I_{c}$ involves solutions of the Usadel equation at all energies. ${ }^{19}$ In order to determine the precise value ${ }^{20}$ of the critical current, we performed a numerical solution of the Usadel equations for the whole range of Matsubara frequencies. In the long-junction limit $\left(\Delta / \epsilon_{c} \rightarrow \infty\right)$, the zero temperature $e R_{\mathrm{N}} I_{c}$ is found to be proportional to $\epsilon_{c}$ :

$$
e R_{\mathrm{N}} I_{c}(T=0)=10.82 \epsilon_{c} .
$$

In this case, the current phase relation is slightly different from a sine and the supercurrent maximum occurs at $\varphi$ $=1.27 \pi / 2$. As compared to previous estimates, ${ }^{11,12}$ the exact numerical prefactor in this formula turns out to be unexpectedly high. This observation is crucial for a quantitative comparison between theory and experiment not only in the case of conventional junctions but also for high- $T_{c}$ S-N-S junctions ${ }^{21}$ or devices involving carbon nanotubes. ${ }^{22}$

Let us briefly consider the short-junction regime $\Delta \ll \epsilon_{c}$, i.e., the case of dirty S-C-S weak links described in Refs. 23 and 24. Our numerical results reproduces quantitatively the behaviors of both the current-phase relation and the zerotemperature critical current: $e R_{N} I_{c} \approx 1.326 \pi \Delta / 2$ at $\varphi$ $=1.25 \pi / 2 \cdot{ }^{23,24}$ This results confirms the precision of our calculation in describing both the long-junction and the shortjunction regimes. Our numerical results for $I_{c}(T=0)$ as a function of the Thouless energy $\epsilon_{c}$ are presented in Fig. 1. It confirms that it is the minimum of the gap $\Delta$ and the Thouless energy $\epsilon_{c}$ that limits the critical current in diffusive S-N-S junctions. At $\epsilon_{c} \simeq \Delta$, the critical current value remains close to the short-junction case.

In the following, we will focus on long junctions $\Delta$ $>\epsilon_{c}$. Figure 2 shows the temperature dependence of the $e R_{\mathrm{N}} I_{c}$ product for various values of the superconducting gap in the long-junction regime. Both axis are given in units of the Thouless energy. The low-temperature part $\left(k_{B} T<5 \epsilon_{c}\right)$

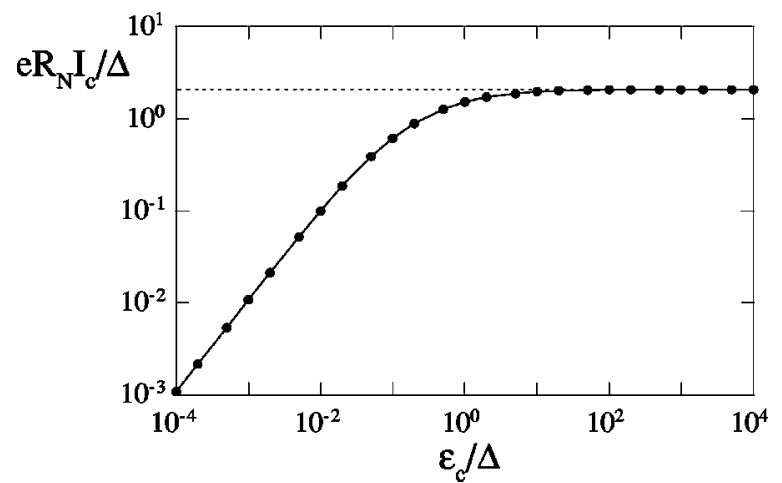

FIG. 1. Calculated dependence of the zero-temperature $e R_{\mathrm{N}} I_{c}$ product in units of $\Delta$ as a function of the ratio $\epsilon_{c} / \Delta . I_{c}$ is the Josephson critical current, $R_{\mathrm{N}}$ the normal-state resistance, $\epsilon_{c}$ is the Thouless energy, and $\Delta$ is the superconducting gap of $\mathrm{S}$. The longjunction regime is on the left part of the graph where $\epsilon_{c}<\Delta$, the short-junction regime is on the right part where $\epsilon_{c}>\Delta$. The dashed line corresponds to the Kulik-Omel'yanchuk formula ${ }^{25}$ at $T=0$.

comes from a numerical solution of the Usadel equation, while the high-temperature part comes from Eq. (1). From this figure, we can see that the characteristic decay temperature for the critical current is a few times the Thouless temperature $\epsilon_{c} / k_{B}$. As soon as $k_{B} T>5 \epsilon_{c}$, the sum in Eq. (1) can be reduced to the first frequency term within a $3 \%$ underestimation. This term corresponds to $\omega_{0}=\pi k_{B} T$ and $L_{\omega_{0}}=L_{T}$. Adding the second term in the summation decreases the error below $0.1 \%$ in the same temperature range.

The universal curve of Fig. 2 for $\Delta / \epsilon_{c} \rightarrow \infty$ is valid only in the case of a very long junction with $\Delta / \epsilon_{c} \gg 100$. It appears as if $\Delta$ is to be compared to the quantity $e R_{N} I_{c}(T=0)$ $\simeq 10 \epsilon_{c}$ in the long-junction limit. In the limit of infinite $\Delta / \epsilon_{c}$, Eq. (1) simplifies to

$$
e R_{\mathrm{N}} I_{c}=\frac{32}{3+2 \sqrt{2}} \epsilon_{c}\left[\frac{L}{L_{T}}\right]^{3} e^{-L / L_{T}}
$$

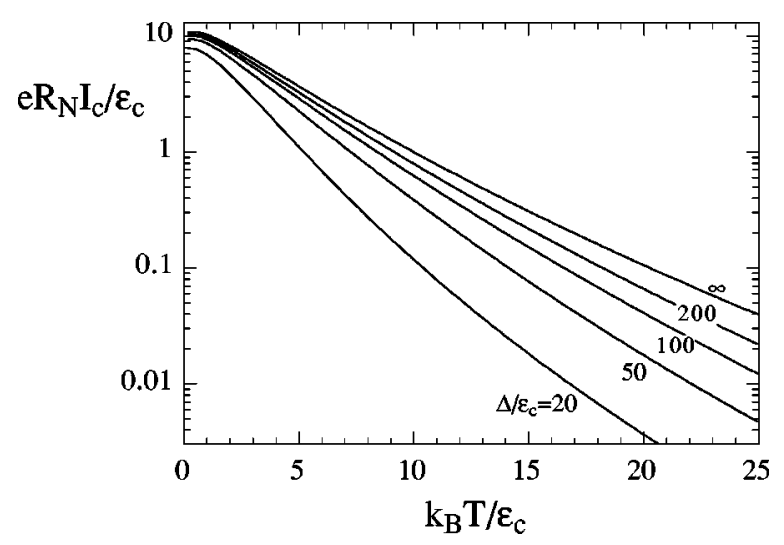

FIG. 2. Calculated temperature dependence of the $e R_{\mathrm{N}} I_{c}$ product. The different curves correspond to various values of the ratio $\Delta / \epsilon_{c}$ in the long-juction regime. The curve for $\Delta / \epsilon_{c} \rightarrow \infty$ is universal in the sense it does not depend on $\Delta$. Note that $k_{B} T / \epsilon_{c}$ $=L^{2} / 2 \pi L_{T}^{2}$. 


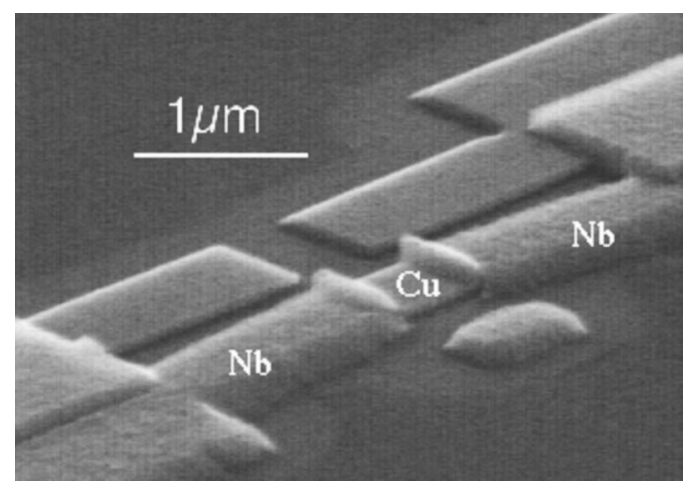

FIG. 3. Oblique micrograph of a typical S-N-S junction made of $\mathrm{a} \mathrm{Cu}$ wire embedded between two $\mathrm{Nb}$ electrodes. The doubling of every structure due to the shadow evaporation is visible. The $\mathrm{Nb}$ electrodes cover the $\mathrm{Cu}$ strip over about $150 \mathrm{~nm}$.

From Eq. (3), one can get the temperature dependence of the critical current: $I_{c} \propto T^{3 / 2} \exp \left(-L / L_{T}\right)$. It has been demonstrated in Ref. 11 that within a limited temperature interval this expression is numerically very close to a simple exponential dependence $I_{c} \propto \exp \left(-L / L_{T}\right)$ with $L_{T} \propto 1 / T$, as one would expect in a ballistic limit. ${ }^{25,26}$ From Fig. 2, the quasiexponential temperature dependence of the critical current is indeed striking. This was the central result of Ref. 10, but was not understood at that time. This coincidence is purely accidental and has no special meaning. ${ }^{11}$ In the lowtemperature limit, the numerical solution can be approximated by $e R_{\mathrm{N}} I_{c} / \epsilon_{c}=a\left(1-b e^{-a \epsilon_{c} / 3.2 k_{B} T}\right)$. The coefficients $a$ and $b$ are 10.82 and 1.30, respectively, in the long-junction limit, i.e., at $\Delta / \epsilon_{c} \rightarrow \infty$.

$\mathrm{S}-\mathrm{N}-\mathrm{S}$ junctions are intrinsically shunted and have negligible internal capacitance, so they are strongly overdamped. Their current-voltage characteristics are hence intrinsically nonhysteretic. The transition from a supercurrent to a voltage state happens at the critical current, but is rounded by finite temperature. ${ }^{27}$ We fabricated $\mathrm{Nb}-\mathrm{Cu}-\mathrm{Nb}$ junctions ${ }^{28}$ with a large conductance so that thermal fluctuations remain small compared to the Josephson energy : $k_{B} T \ll \hbar I_{c}(T) / 2 e$ even at high temperature near the critical temperature of $\mathrm{Nb}$. This ensures a well-defined critical current up to the critical temperature of $\mathrm{Nb}$. Effects of environmental fluctuations known from mesoscopic tunnel junctions, ${ }^{29}$ which are intrinsically underdamped, are absent.
We benefited from a trilayer stencil mask technology $\mathrm{y}^{30}$ making use of a thermostable resist that does not outgas during $\mathrm{Nb}$ evaporation. Thus we were able to routinely obtain a superconducting critical temperature as high as $8.1 \mathrm{~K}$ for the $\mathrm{Nb}$ electrodes. We performed successive shadow evaporations of $\mathrm{Cu}$ and $\mathrm{Nb}$ at different angles through the silicon stencil layer in an ultra-high vacuum chamber, followed by a liftoff. Figure 3 shows a typical sample. We studied a single sample $(a)$ plus five different samples evaporated on the same substrate $(b, c, d, e$, and $f)$. Table I lists the main physical parameters for these samples. The $\mathrm{Cu}$ metallic strips are $600 \mathrm{~nm}$ wide and $100 \mathrm{~nm}$ thick. The $\mathrm{Nb}$ superconducting electrodes are $800 \mathrm{~nm}$ wide and $200 \mathrm{~nm}$ thick, except for sample $a$ where it is $100 \mathrm{~nm}$. The length $L$ of the metallic island was varied between 700 and $1000 \mathrm{~nm}$, corresponding to a separation length $d_{\mathrm{Nb}}$ between $\mathrm{Nb}$ electrodes varying between 370 and $700 \mathrm{~nm}$. For all samples, the calculated Thouless energy $\hbar D / L^{2}$ is therefore significantly smaller than the gap $\Delta$.

The normal-state resistance $R_{\mathrm{N}}$ cannot be directly measured at temperature above $T_{c}$ since the resistance of the $\mathrm{Nb}$ electrodes is measured in series. We found that the finite-bias resistance $\left(e V \simeq \epsilon_{c}\right)$ varied by about $10 \%$ between $2 \mathrm{~K}$ and $8 \mathrm{~K}$ due to the proximity effect on the conductance. We took for the normal-state resistance $R_{\mathrm{N}}$ the resistance at $T$ $=6 \mathrm{~K}$ for a better agreement with theory. It is a relatively high temperature since $k_{B} T>15 \epsilon_{c}$ for every sample then. Using $L$ for the $\mathrm{Cu}$ length, we obtain a $\mathrm{Cu}$ resistivity $\rho=1.1 \times 10^{-8} \Omega \mathrm{m}$ for samples $b$ to $f$ and $\rho=1.5$ $\times 10^{-8} \Omega \mathrm{m}$ for sample $a$.

We measured the critical current of samples $a$ to $f$ at temperatures down to $300 \mathrm{mK}$. Our procedure consists of sweeping the bias current while measuring the differential resistance $d V / d I$. We define the experimental critical current as the current where the differential resistance reaches $R_{\mathrm{N}} / 2$. With this criteria, the experimental uncertainty is estimated below $0.5 \%$ at $T=2 \mathrm{~K}, 5 \%$ around $T=4 \mathrm{~K}$ and $100 \%$ at $7 \mathrm{~K}$. Figure 4 shows the data for three samples. The measured $e R_{\mathrm{N}} I_{c} / \epsilon_{c}$ plotted as a function of the reduced temperature $k_{B} T / \epsilon_{c}$ show a large decrease over more than two decades. For each sample, we fitted the data to the theoretical prediction with only one fitting parameter, the Thouless energy. The zero-temperature superconducting gap $\Delta$ was calculated from the measured critical temperature of $\mathrm{Nb}$ using

TABLE I. Parameters of the different samples studied. $L$ is the full length of $\mathrm{Cu}$ strip, while $d_{\mathrm{Nb}}$ is the $\mathrm{Nb}$ electrodes separation and $w$ is the $\mathrm{Cu}$ strip width. The Thouless energy $\boldsymbol{\epsilon}_{c}$ is derived from the fit of the experimental data to the theoretical prediction (see Fig. 4).

\begin{tabular}{lccccccccc}
\hline \hline$\#$ & $L$ & $d_{\mathrm{Nb}}$ & $w$ & $R_{\mathrm{N}, 6 \mathrm{~K}}$ & $\begin{array}{c}D \\
\left(\mathrm{~cm}^{2} / \mathrm{s}\right)\end{array}$ & $\begin{array}{c}\hbar D / L^{2} \\
(\mu \mathrm{eV})\end{array}$ & $\epsilon_{c}$ & $\Delta / \epsilon_{c}$ & $\frac{e R_{\mathrm{N}} I_{c}}{\varepsilon_{c}}(T=0)$ \\
\hline$a$ & 1000 & 600 & 600 & 0.260 & 200 & 13 & 14.3 & 70 & 8.91 \\
$b$ & 1010 & 680 & 580 & 0.173 & 300 & 20 & 18.6 & 70 & 8.99 \\
$c$ & 910 & 570 & 590 & 0.179 & 260 & 22 & 21.7 & 60 & 8.83 \\
$d$ & 800 & 470 & 580 & 0.183 & 230 & 25 & 25.4 & 51 & 8.64 \\
$e$ & 800 & 476 & 590 & 0.169 & 250 & 26 & 26.1 & 50 & 8.62 \\
$f$ & 710 & 370 & 580 & 0.152 & 250 & 34 & 33.5 & 39 & 8.32 \\
\hline \hline
\end{tabular}




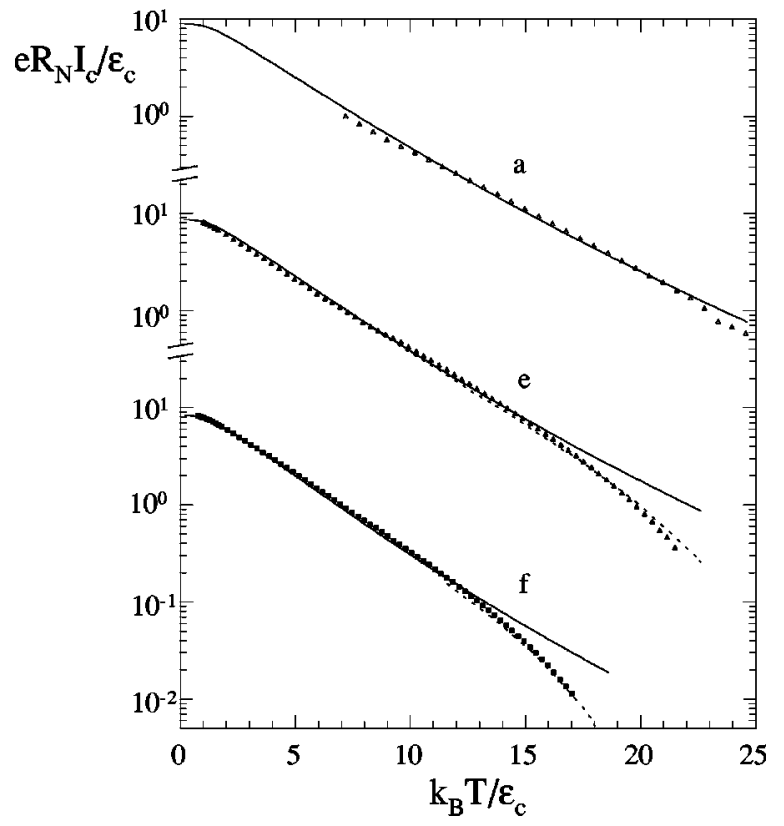

FIG. 4. Temperature dependence of the measured $e R_{\mathrm{N}} I_{c}$ product of samples $a, e$, and $f$ together with the theoretical fits assuming a temperature-independent gap (full line) and a gap following a BCS temperature dependence with $T_{c}=7.5 \mathrm{~K}$ (dashed line). The only adjustment parameter is the Thouless energy $\epsilon_{c}$ of each sample. For a description of the sample parameters, see Table I.

$2 \Delta=3.8 k_{B} T_{c} \cdot{ }^{31}$ This gives $\Delta=1.3 \mathrm{meV}$ for all samples except sample $a$ for which $\Delta=1 \mathrm{meV}$. We used both a fixed gap equal to the zero-temperature value and a gap $\Delta(T)$ following the BCS temperature dependence, but with a slightly reduced critical temperature $T_{c}=7.5 \mathrm{~K}$. For samples $e, f$ and at high temperature, it appears necessary to take into account the temperature dependence of the gap. In this case, the agreement between theory and experiment is excellent. The fit is very sensitive to the chosen value of the Thouless energy. We would like to stress that for each sample the horizontal and vertical axis are normalized to the same Thouless energy $\epsilon_{c}$. Each such value is found to be very close to the Thouless energy calculated from the full length $L$ of the $\mathrm{Cu}$ strip; see Table I.
In Fig. 4, the critical current of sample $f$ shows the onset of the saturation regime. At $T=300 \mathrm{mK}$ the adjusted critical current $e R_{\mathrm{N}} I_{c}$ reaches up to $8.2 \epsilon_{c}$. This number is close to the theoretical value $8.79 \epsilon_{c}$ for sample $f$ at $T=0$. This result discards an interpretation of our data within the GinzburgLandau theory of Ref. 12, which predicts a maximum $e R_{\mathrm{N}} I_{c} / \epsilon_{c}$ of about 1 .

In Ref. 10, an array of S-N-S junctions was made of a long $\mathrm{N}$-metal wire periodically in contact with a series of superconducting islands. A good fit between the data and theory was shown in Ref. 11, but with the introduction of a strong reduction of the effective area. This may be attributed to the periodic and lateral characters of this type of samples.

Our calculation assumes perfectly transmitting interfaces with zero boundary resistance. In fact, it is sufficient that the barrier-equivalent length ${ }^{32} L_{t}=l_{e} / t$ is much smaller than the sample length. As an example, this condition means an interface transparency $t>0.1$ for sample b. In the case of $\mathrm{Nb}$ $\mathrm{Cu}-\mathrm{Nb}$ samples fabricated through a two-lithography-step process including Ar etching, ${ }^{33}$ we found a critical current with a reduced magnitude, presumably due to a slightly degraded interface. The critical current in S-N-S junctions with partially transparent interfaces was discussed in Ref. 34. The predicted behavior features a different temperature dependence for the critical current. Nevertheless, the measured temperature dependence remained consistent with theory assuming a perfect interface. Only a reduction prefactor had to be introduced. This observation could hint at the fact that interface barriers are very inhomogenous and the current is carried through a few highly conducting pinholes.

In summary, we discussed the Josephson critical current of diffusive S-N-S junctions. This study provides a simple and reliable formulation that enables the practical determination of the equilibrium critical current. We studied the critical current of a set of samples with different junction lengths and found excellent agreement between our data and the predictions of quasiclassical theory.

We acknowledge discussion and financial support in the EU-TMR network "Dynamics of superconducting circuits" as well as support from the DFG through SFB 195 and GK 284. We thank A. Golubov, T.T. Heikkilä, D. Mailly, N. Moussy, and P. Paniez for discussions.
${ }^{1}$ J. Clarke, Proc. R. Soc. London, Ser. A 308, 447 (1969).

${ }^{2}$ J.G. Shepherd, Proc. R. Soc. London, Ser. A 326, 421 (1972).

${ }^{3}$ P.G. de Gennes, Rev. Mod. Phys. 36, 225 (1964).

${ }^{4}$ H.J. Fink, Phys. Rev. B 14, 1028 (1976).

${ }^{5}$ J. Warlaumont, J.C. Brown, and R.A. Buhrman, Appl. Phys. Lett. 34, 415 (1979).

${ }^{6}$ R.B. van Dover, A. de Lozanne, and M.R. Beasley, J. Appl. Phys. 52, 7327 (1981).

${ }^{7}$ K.K. Likharev, Sov. Tech. Phys. Lett. 2, 12 (1976).

${ }^{8}$ K.D. Usadel, Phys. Rev. Lett. 25, 507 (1970).

${ }^{9}$ A.D. Zaikin and G.F. Zharkov, Sov. J. Low Temp. Phys. 7, 184 (1981). In Eq. 14 and 15 of that paper, the prefactor 128 refers to the case where $R_{N}$ is the resistance per spin. It should be re- placed by 64 if one identifies $R_{N}$ with the full resistance.

${ }^{10}$ H. Courtois, Ph. Gandit, and B. Pannetier, Phys. Rev. B 52, 1162 (1995).

${ }^{11}$ F.K. Wilhelm, A.D. Zaikin, and G. Schön, J. Low Temp. Phys. 106, 305 (1997).

${ }^{12}$ H.J. Fink, Phys. Rev. B 56, 2732 (1997).

${ }^{13}$ See the special issue of Superlattices Microstruct. 25 (5/6), 6271288 (1999).

${ }^{14}$ H. Courtois, Ph. Gandit, D. Mailly, and B. Pannetier, Phys. Rev. Lett. 76, 130 (1996).

${ }^{15}$ B. Pannetier and H. Courtois, J. Low Temp. Phys. 118, 599 (2000).

${ }^{16}$ J.J.A. Baselmans, A.F. Morpurgo, B.J. van Wees, and T.M. Klap- 
wijk, Nature (London) 397, 43 (1999).

${ }^{17}$ F.K. Wilhelm, G. Schön, and A.D. Zaikin, Phys. Rev. Lett. 81, 1682 (1998).

${ }^{18}$ J. Kutchinsky, R. Taboryski, C.B. Sørensen, J.B. Hansen, and P.E. Lindelof, Phys. Rev. Lett. 83, 4856 (1999).

${ }^{19}$ W. Belzig, F. K. Wilhelm, C. Bruder, G. Schön, and A. D. Zaikin, in Ref. 13.

${ }^{20}$ In the long-junction regime $\Delta>\epsilon_{\mathrm{c}}$, the extrapolation of low-(Ref. 11) or high-(Ref. 12) energy dependencies provides a reasonable parameter dependence, but fails to predict the correct prefactor.

${ }^{21}$ K.A. Delin and A.W. Kleinsasser, Supercond. Sci. Technol. 9, 227 (1996).

${ }^{22}$ A.Yu. Kasumov, R. Deblock, M. Kociak, B. Reulet, H. Bouchiat, I. I. Khodos, Yu. B. Gorbatov, V. T. Volkov, C. Journet, and M. Burghard, Science 284, 1508 (1999).

${ }^{23}$ I.O. Kulik and A.N. Omel'yanchuk, Sov. J. Low Temp. Phys. 4, 142 (1978).

${ }^{24}$ A.D. Zaikin and S.V. Panyukov, Zh. Eksp. Teor. Fiz. 89, 242
(1985) [Sov. Phys. JETP 62, 137 (1985)].

${ }^{25}$ I.O. Kulik, Zh. Éksp. Teor. Fiz. 57, 1745 (1969) [Sov. Phys. JETP 30, 944 (1970)].

${ }^{26}$ C. Ishii, Prog. Theor. Phys. 14, 1525 (1970).

${ }^{27}$ V. Ambegaokar and B.I. Halperin, Phys. Rev. Lett. 22, 1364 (1969).

${ }^{28}$ F.K. Wilhelm, G. Schön, A.D. Zaikin, A.A. Golubov, P. Dubos, H. Courtois, and B. Pannetier, Physica B 284-288, 1836 (2000).

${ }^{29}$ D. Vion, M. Götz, P. Joyez, D. Estève, and M.H. Devoret, Phys. Rev. Lett. 77, 3435 (1996).

${ }^{30}$ P. Dubos, P. Charlat, Th. Crozes, P. Paniez, and B. Pannetier, J. Vac. Sci. Technol. B 18, 122 (2000).

${ }^{31}$ N. Ashcroft and N. Mermin, Solid State Physics (Holt-Saunders, Orlando, FL, 1976).

${ }^{32}$ F. Zhou, B. Spivak, and A. Zyuzin, Phys. Rev. B 52, 4467 (1995).

${ }^{33}$ P. Dubos and D. Mailly (unpublished).

${ }^{34}$ M.Yu. Kuprianov and V.F. Lukichev, Zh. Éksp. Teor. Fiz. 94, 139 (1988) [Sov. Phys. JETP 67, 1163 (1988)]. 